02

\title{
Кристаллическая структура и люминесценция комплекса $\left[\mathrm{DyCl}_{2}\left(\mathrm{H}_{2} \mathrm{O}\right)_{6}\right] \mathrm{Cl}$
}

\author{
(С) Б.В. Буквецкий, А.Г. Мирочник , П.А. Жихарева \\ Институт химии Дальневосточного отделения РАН, \\ 690022 Владивосток, Россия \\ ๑e-mail: mirochnik@ich.dvo.ru
}

Поступила в редакцию 01.11.2019 г.

В окончательной редакции 03.12.2019 г.

Принята к публикации 06.12.2019 г.

Методом рентгеноструктурного анализа определена кристаллическая структура комплекса $\left[\mathrm{DyCl}_{2}\left(\mathrm{H}_{2} \mathrm{O}\right)_{6}\right] \mathrm{Cl}$. Кристаллы построены из комплексных катионов $\left[\mathrm{DyCl}_{2}\left(\mathrm{H}_{2} \mathrm{O}\right)_{6}\right]^{+}$и внешнесферных $\mathrm{Cl}^{-1}$-ионов. Структура представлена обособленными комплексами $\left[\mathrm{DyCl}_{2}\left(\mathrm{H}_{2} \mathrm{O}\right)_{6}\right]$, связанными густой сеткой водородных связей типа $\mathrm{O}-\mathrm{H} \ldots \mathrm{Cl}$. Координационный полиэдр Dy (III) с координационным числом 8 представлен слабо искаженной квадратной антипризмой. Представлены результаты исследования люминесцентных свойств комплекса.

Ключевые слова: диспрозий(III), кристаллическая структура, люминесценция.

DOI: 10.21883/OS.2020.03.49060.295-19

Уникальные люминесцентные свойства соединений лантанидов (узкие, практически монохроматические полосы эмиссии, большие стоксовы сдвиги и фотостабильность) делают их привлекательными для разработки высокочувствительных хемо- и триболюминесцентных сенсорных устройств [1-3]. Комплексные соединения лантанидов интересны своими уникальными люминесцентными и фотохимическими свойствами, они могут быть использованы в качестве новых оптических материалов (светотрансформирующие полимерные материалы, эффективные люминофоры в современных люминесцентных лампах, лазерные системы, флуоресцентные метки для биоанализа, органические светодиоды (OLEDs) и др.) [4,5]. Комплексные соединения диспрозия(III) (электронная конфигурация $4 f^{9}$ ), обладающие интенсивной люминесценцией в видимом и ИК диапазонах, перспективны для разработки оптических усилителей [6-8], люминофоров белого свечения [9-14]. В продолжение наших работ по изучению взаимосвязи молекулярного строения и люминесцентных свойств комплексов Dy(III) [15] в настоящей работе нами исследован комплекс $\left[\mathrm{DyCl}_{2}\left(\mathrm{H}_{2} \mathrm{O}\right)_{6}\right] \mathrm{Cl}$.

\section{Экспериментальная часть}

Комплекс получен перекристаллизацией $\mathrm{DyCl}_{3} 6 \mathrm{H}_{2} \mathrm{O}$ (Новосибирский завод редких металлов) в водноэтанольном растворе. Соединение представляет собой мелкокристаллический порошок белого цвета, гигроскопичен, хорошо растворим в воде и спирте, нерастворим в эфире и бензоле.

Рентгеноструктурное исследование проводили с помощью системы Карра APEXII CCD $\left(\mathrm{MoK}_{\alpha}\right.$-излучение, графитовый монохроматор) с использованием программ [16]. Структура определялась прямым методом с использованием программ [17]. Положения атомов водорода определены из синтезов электронной плотности и уточнены с использованием модели „наездника“. CIF-файл, содержащий полную информацию об изучаемой структуре, депонирован в CCDC (Кембриджский центр кристаллографических данных) под номером 1962351 и может быть получен по запросу www.ccdc.cam.ac.uk/data_request/cif. Основные кристаллографические параметры исследуемого образца, харак-

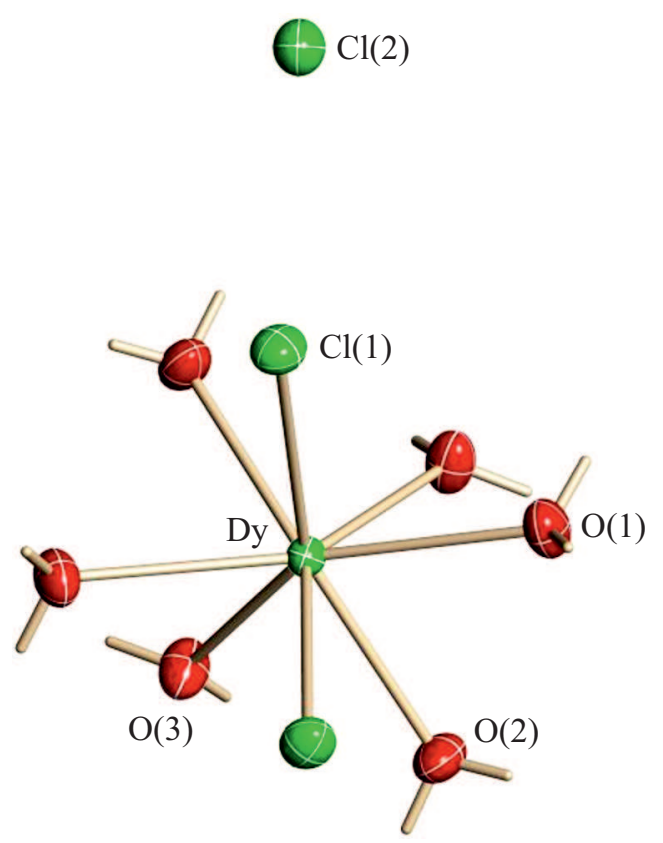

Pис. 1. Молекулярная структура комплекса $\left[\mathrm{DyCl}_{2}\left(\mathrm{H}_{2} \mathrm{O}\right)_{6}\right] \mathrm{Cl}$. 


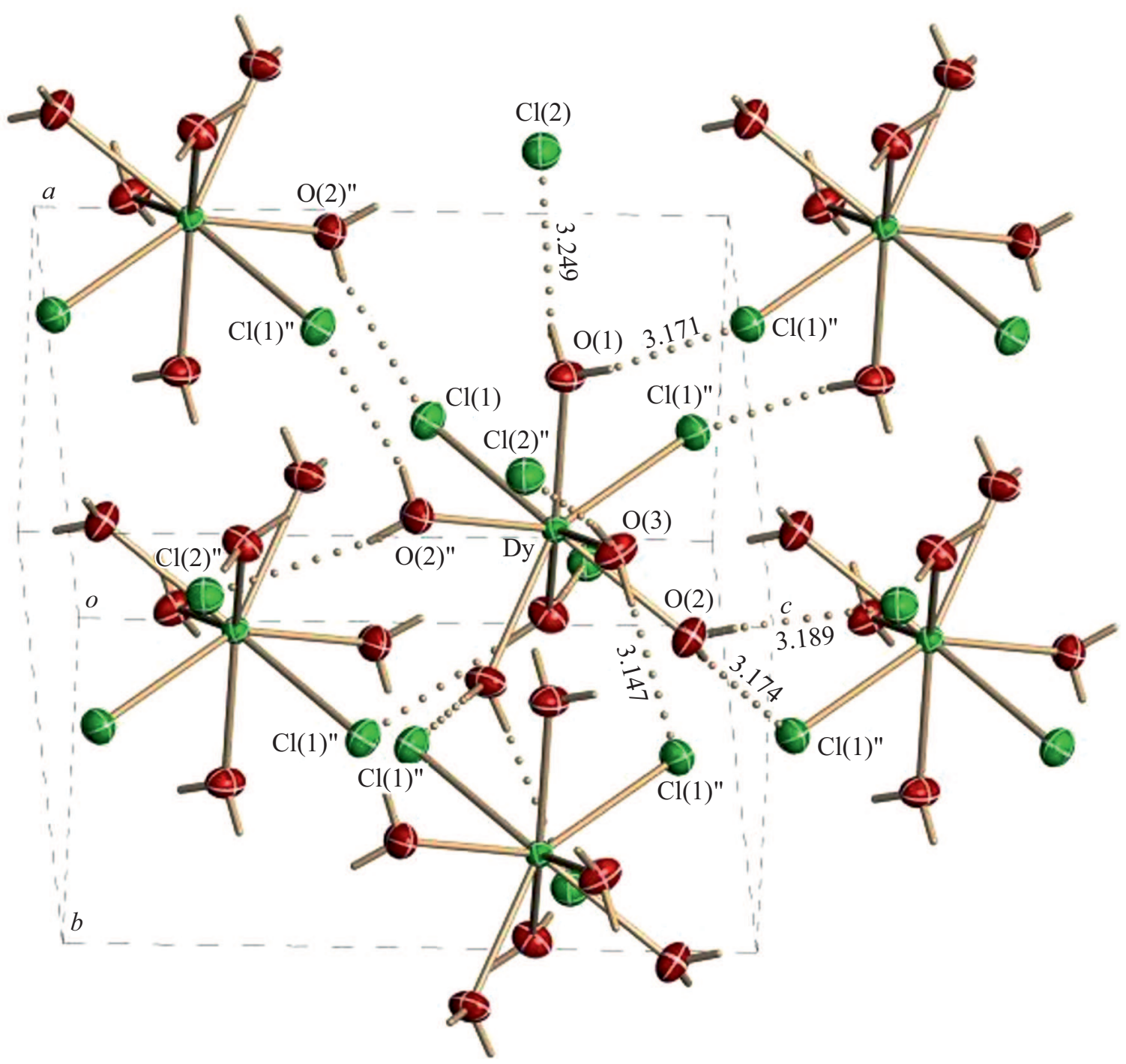

Рис. 2. Кристаллическая структура комплекса $\left[\mathrm{DyCl}_{2}\left(\mathrm{H}_{2} \mathrm{O}\right)_{6}\right] \mathrm{Cl}$.

теристики рентгеновского дифракционного эксперимента и детали уточнения модели структуры приведены в табл. 1, а основные межатомные расстояния и валентные углы - в табл. 2.

Спектры люминесценции комплекса измерены на спектрофлуориметре RF5301 (Шимадзу) при 300 К. Кинетика люминесценции регистрировались на спектрофлуориметре Horiba Fluorolog 3 (Япония) при 300 и $77 \mathrm{~K}$.

\section{Результаты и обсуждение}

Молекулярная структура соединения $\left[\mathrm{DyCl}_{2}\left(\mathrm{H}_{2} \mathrm{O}\right)_{6}\right] \mathrm{Cl}$ представлена на рис. 1. Кристаллы $\left[\mathrm{DyCl}_{2}\left(\mathrm{H}_{2} \mathrm{O}\right)_{6}\right] \mathrm{Cl}$ построены из обособленных комплексных $\left[\mathrm{DyCl}_{2}\left(\mathrm{H}_{2} \mathrm{O}\right)_{6}\right]^{+}$катионов и внешнесферных $\mathrm{Cl}^{-1}$-ионов. Ближайшие расстояния от люминесцентного центра Dy(III) до атомов водорода молекул воды находятся в пределах
2.800-2.89 А(рис. 1). Два атома хлора и шесть атомов кислорода молекул воды образуют вокруг атома Dy слабо искаженную квадратную антипризму.

Диспрозиевые комплексы жестко увязаны в трехмерный каркас густой сеткой водородных связей как непосредственно между комплексами типа О-Н . . Сl с длинами от 3.147 до $3.299 \AA$, так и через посредство $\mathrm{Cl}^{-1}$ ионов с длинами от 3.180 до $3.299 \AA$ (рис. 2). Следует отметить, что в отличие от исследуемого нами в [15] комплекса $\left[\mathrm{Dy}\left(\mathrm{NO}_{3}\right)_{2}[\mathrm{HMPA})_{4}\right]\left(\mathrm{NO}_{3}\right)$ для $\left[\mathrm{DyCl}_{2}\left(\mathrm{H}_{2} \mathrm{O}\right)_{6}\right] \mathrm{Cl}$ не удалось зарегистрировать ИК люминесценцию. Повидимому, наличие густой сетки водородных связей в структуре приводит к эффективной диссипации энергии электронного возбуждения в ИК диапазоне.

На рис. 3 и 4 показаны спектры люминесценции и возбуждения люминесценции комплекса $\left[\mathrm{DyCl}_{2}\left(\mathrm{H}_{2} \mathrm{O}\right)_{6}\right] \mathrm{Cl}$ соответственно. Исследуемый комплекс $\left[\mathrm{DyCl}_{2}\left(\mathrm{H}_{2} \mathrm{O}\right)_{6}\right] \mathrm{Cl}$ обладает люминесценцией (по- 
Таблица 1. Кристаллографические данные, характеристики рентгендифракционного эксперимента и детали уточнения структуры $\left[\mathrm{DyCl}_{2}\left(\mathrm{H}_{2} \mathrm{O}\right)_{6}\right] \mathrm{Cl}$

\begin{tabular}{|c|c|}
\hline Параметр & Значение \\
\hline Формула & $\mathrm{H}_{24} \mathrm{Dy}_{2} \mathrm{Cl}_{6} \mathrm{O}_{12}$ \\
\hline Молекулярная масса & 1602.34 \\
\hline Температура & $296(2) \mathrm{K}$ \\
\hline Длина волны & $\operatorname{MoK}_{\alpha}(0.71073 \AA)$ \\
\hline Пространственная группа & $P 2 / n$ \\
\hline$a, \AA$ & $7.8757(1)$ \\
\hline$b, \AA$ & $6.4951(1)$ \\
\hline$c, \AA$ & $9.6031(1)$ \\
\hline$\beta, \operatorname{deg}$ & $93.704(1)$ \\
\hline $\mathrm{Z}$ & 2 \\
\hline$\rho_{\text {выч. }}, \mathrm{g} / \mathrm{cm}^{3}$ & 5.428 \\
\hline$\mu, \mathrm{mm}^{-1}$ & 5.171 \\
\hline$F(000)$ & 814 \\
\hline Размер кристалла, mm & $0.14 \times 0.13 \times 0.04$ \\
\hline Область сбора данных по $\theta$ & $3.46^{\circ}-39.54^{\circ}$ \\
\hline $\begin{array}{l}\text { Интервалы индексов } \\
\text { отражений }\end{array}$ & $\begin{array}{l}-14 \leq h 10,-11 \leq k \leq 11 \\
-17 \leq l \leq 17\end{array}$ \\
\hline Измерено отражений & 10220 \\
\hline Независимых отражений & $2923\left(R_{\text {int }}=0.0236\right)$ \\
\hline Отражений с $I>2 \sigma(\mathrm{I})$ & 2704 \\
\hline Комплексность по $\theta$ до $39.54^{\circ}$ & $98.7 \%$ \\
\hline Поглощение & $\begin{array}{l}\text { По индексам огранки } \\
\text { кристалла }\end{array}$ \\
\hline Метод уточнения & Полноматричный МНК по $F^{2}$ \\
\hline Переменных уточнения & 72 \\
\hline $\mathrm{S}$ & 1.049 \\
\hline$R$-факторы по $I>2 \sigma(\mathrm{I})$ & $R_{1}=0.0166, w R 2=0.0296$ \\
\hline $\begin{array}{l}R \text {-факторы по всем } \\
\text { отражениям }\end{array}$ & $R_{1}=0.0197, w R_{2}=0.0302$ \\
\hline $\begin{array}{l}\text { Абсолютный структурный } \\
\text { параметр }\end{array}$ & $0.507(7)$ \\
\hline $\begin{array}{l}\text { Остаточная эл. пл. } \min / \max ), \\
\text { e/A }\end{array}$ & $-0.838 / 0.517$ \\
\hline
\end{tabular}

лосы на длинах волн 490, 572 и $680 \mathrm{~nm}$ (рис. 3)), что соответствует электронным переходам Dy ${ }^{3+}{ }^{4} F_{9 / 2} \rightarrow{ }^{6} H_{J}$ $(J=15 / 2,13 / 2,11 / 2)$ соответственно. По данным анализа спектра возбуждения люминесценции комплек-
Таблица 2. Основные длины связей и валентные углы в структуре $\left[\mathrm{DyCl}_{2}\left(\mathrm{H}_{2} \mathrm{O}\right)_{6}\right] \mathrm{Cl}$

\begin{tabular}{l|l|l|l}
\hline Связь & $d, \AA$ & Угол & $\varphi, \operatorname{deg}$ \\
\hline Dy-O(3) & $2.3508(11)$ & $\mathrm{O}(3)-\mathrm{Dy}-\mathrm{O}(3) \# 1$ & $83.46(6)$ \\
\hline $\mathrm{Dy}-\mathrm{O}(2)$ & $2.3810(10)$ & $\mathrm{O}(3)-\mathrm{Dy}-\mathrm{O}(2) \# 1$ & $75.68(4)$ \\
\hline $\mathrm{Dy}-\mathrm{O}(1)$ & $2.3845(10)$ & $\mathrm{O}(3)-\mathrm{Dy}-\mathrm{O}(2)$ & $69.52(4)$ \\
\hline Dy-Cl(1) & $2.7478(3)$ & $\mathrm{O}(3)-\mathrm{Dy}-\mathrm{O}(1) \# 1$ & $70.57(4)$ \\
\hline & & $\mathrm{O}(2)-\mathrm{Dy}-\mathrm{O}(1)$ & $73.02(4)$ \\
\hline & & $\mathrm{O}(2) \# 1-\mathrm{Dy}-\mathrm{Cl}(1)$ & $76.20(3)$ \\
\hline & & $\mathrm{O}(1) \# 1-\mathrm{Dy}-\mathrm{Cl}(1)$ & $78.85(3)$ \\
\hline & & $\mathrm{O}(1)-\mathrm{Dy}-\mathrm{Cl}(1)$ & $77.12(3)$ \\
\hline & & $\mathrm{Cl}(1)-\mathrm{Dy}-\mathrm{Cl}(1) \# 1$ & $83.696(15)$
\end{tabular}

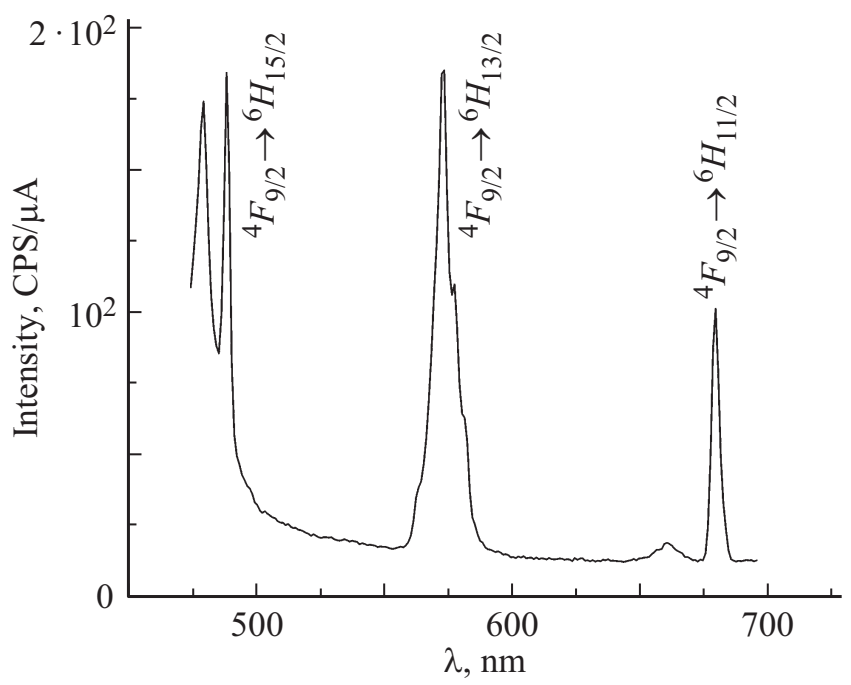

Pис. 3. Спектр люминесценции комплекса $\left[\mathrm{DyCl}_{2}\left(\mathrm{H}_{2} \mathrm{O}\right)_{6}\right] \mathrm{Cl}$ $\left(\lambda_{\mathrm{ex}}=453 \mathrm{~nm}\right)$.

са (рис. 4) свечение обусловлено $f-f$-переходами $\mathrm{Dy}^{3+}$ (переходы ${ }^{6} P_{J} \leftarrow{ }^{4} H_{15 / 2} ; J=3 / 2,5 / 2,7 / 2 ; 324$, $338,350 \mathrm{~nm}),{ }^{4} M_{15 / 2} \leftarrow{ }^{4} H_{15 / 2}(362 \mathrm{~nm}),{ }^{4} H_{13 / 2} \leftarrow{ }^{4} H_{15 / 2}$ $(388 \mathrm{~nm}), \quad{ }^{4} G_{11 / 2} \leftarrow{ }^{4} H_{15 / 2} \quad(430 \mathrm{~nm}), \quad{ }^{4} I_{15 / 2} \leftarrow{ }^{4} H_{15 / 2}$ $(452 \mathrm{~nm})$. Кинетику люминесценции $\mathrm{Dy}^{3+}$ (уровень $\left.{ }^{4} F_{9 / 2}\right)$ комплекса $\left[\mathrm{DyCl}_{2}\left(\mathrm{H}_{2} \mathrm{O}\right)_{6}\right] \mathrm{Cl}$ измеряли при 300 и $77 \mathrm{~K}$ с использованием длины волны возбуждения $453 \mathrm{~nm}$ (рис. 5). Кинетика люминесценции описывается моноэкспоненциальной функцией, что согласуется с наличием в комплексе одного люминесцентного центра. Соответствующее время жизни возбужденного состояния Dy $\left({ }^{4} F_{9 / 2}\right)$ для комплекса $\left[\mathrm{DyCl}_{2}\left(\mathrm{H}_{2} \mathrm{O}\right)_{6}\right] \mathrm{Cl}$ составляет $2.27 \pm 0.05 \mu \mathrm{s}(300 \mathrm{~K})$ и $2.42 \pm 0.05 \mu \mathrm{s}(77 \mathrm{~K})$ соответственно.

Методом рентгеноструктурного анализа определена атомная структура кристаллов комплекса 


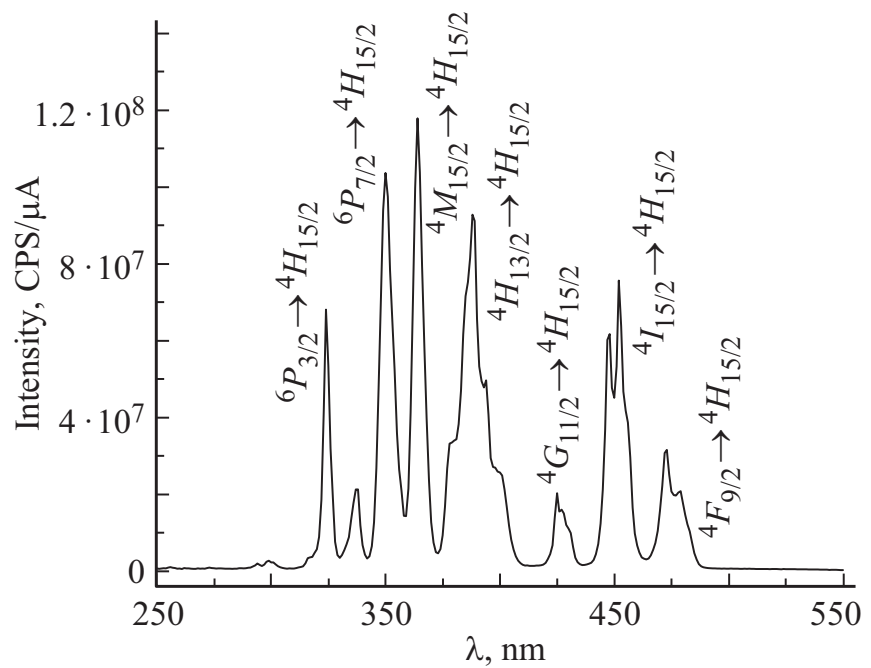

Рис. 4. Спектр возбуждения люминесценции комплекса $\left[\mathrm{DyCl}_{2}\left(\mathrm{H}_{2} \mathrm{O}\right)_{6}\right] \mathrm{Cl}\left(\lambda_{\text {reg }}=573 \mathrm{~nm}\right)$.

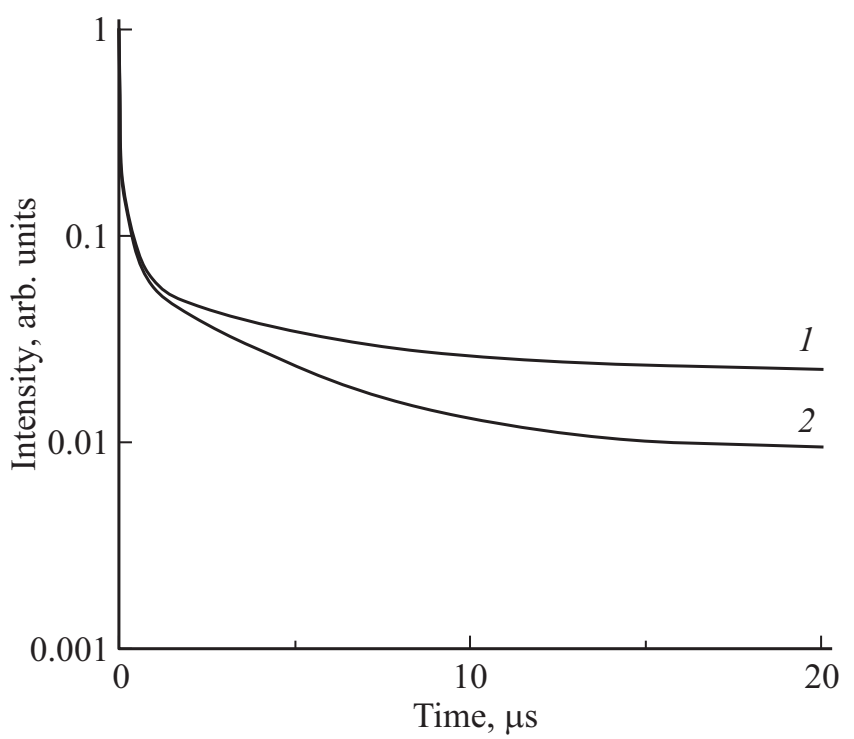

Рис. 5. Кинетика люминесценции $\left[\mathrm{DyCl}_{2}\left(\mathrm{H}_{2} \mathrm{O}\right)_{6}\right] \mathrm{Cl}$ при $300 \mathrm{~K}$ $(1-\tau=2.27 \mu \mathrm{s})$ и при $77 \mathrm{~K}(2-\tau=2.42 \mu \mathrm{s})\left(\lambda_{\mathrm{ex}}=453 \mathrm{~nm}\right.$, $\left.\lambda_{\text {reg }}=573 \mathrm{~nm}\right)$.

$\left[\mathrm{DyCl}_{2}\left(\mathrm{H}_{2} \mathrm{O}\right)_{6}\right] \mathrm{Cl}$, которая представлена изолированными комплексными катионами состава $\left[\mathrm{DyCl}_{2}\left(\mathrm{H}_{2} \mathrm{O}\right)_{6}\right]^{+}$ и внешнесферными $\mathrm{Cl}^{-1}$-ионами. Отсутствие ИК люминесценции в комплексе обусловлено наличием густой сетки водородных связей в структуре, что приводит к эффективной диссипации энергии электронного возбуждения в ИК диапазоне.

\section{Список литературы}

[1] Olawale D.O., Okoli O.O.I., Fontenot R.S., Hollerman W.A. (Eds.) Triboluminescence. Theory, Synthesis, and Application. Springer, Switzerland. 2016. Р. 39-63. [Электронный ресурс] Режим доступа: https:/www.springer.com/la/book/9783319388410.

[2] Hao Z.M., Yang G.C., Song X.Z., Zhu M., Meng X., Zhao S.N., Song S.Y., Zhang H.J. // J. Mater. Chem. A. 2014. V. 2. P. 237. doi 10.1039/C3TA13179C

[3] Mirochnik A.G., Petrochenkova N.V., Shishov A.S., Bukvetskii B.V., Emelina T.B., Sergeev A.A., Voznesenskii S.S. // Spectrochim. Acta A. 2016. V. 155. P. 111. doi 10.1016/j.saa.2015.11.004

[4] Bunzli J-C.G. // Chem. Rev. 2010. V. 110. P. 2729. doi 10.1021/cr900362e

[5] Bunzli J-C.G., Eliseeva S.V. // Chem. Sci. 2013. V. 4. P. 1939. doi 10.1039/C3SC22126A

[6] Hommerich U., Nyein E., Freeman J.A., Amedzake P., Trivedi S.B., Zavada J.M.J. // J. Cryst. Growth. 2006. V. 287. P. 230. doi 10.1016/j.jcrysgro.2005.11.019

[7] Feng J., Zhou L., Song S.Y., Li Z.F., Fan W.Q., Sun L.N., Yu Y.N., Zhang H.J. // Dalton Trans. 2009. P. 6593. doi $10.1039 / \mathrm{b} 906419 \mathrm{~b}$

[8] Wie K., Machewirth D.P., Wenzal J., Snitzer E., Sigel G.H. // Opt. Lett. 1994. V. 19. P. 904. doi 10.1364/OL.19.000904

[9] Huang X.D., Xu Y., Fan K., Bao S.S., Kurmoo M., Zheng L.M. // Angew. Chem. 2018. V. 57. P. 8577. doi 10.1002/anie.201804102

[10] Venkataravanappa M., Basavaraj R.B., Darshan G.P., Prasad B.D.E., Sharma S.C., Prabha P.H., Nagabhushana H. // J. Rare Earths. 2018. V. 36. P. 690. doi 10.1016/j.jre.2017.11.013

[11] Dar W.A., Ahmed Z., Iftikhar K. // J. Photochem. Photobiol. A. 2018. V. 356. P. 502. doi 10.1016/j.jphotochem.2017.12.017

[12] Wang J.H., Chorazy S., Nakabayashi K., Sieklucka B., Ohkoshi S. // J. Mater. Chem. C. 2018. V. 6. P. 473. doi 10.1039/C7TC03963H

[13] Su Q., Pei Z., Chi L., Zhang H., Zhang Z., Zou F.J. // J. Alloys Compd. 1993. V. 192. P. 25. doi 10.1016/0925-8388(93)90174-L

[14] Liu X., Liu Y., Yan D., Zhu H., Liu C., Xu C., Liu Y., Wang X. // J. Mater. Chem. 2012. V. 22. P. 16839. doi 10.1039/C2JM32741D

[15] Буквечкий Б.В., Мирочник А.Г., Жихарева П.А. // Опт. и спектр. 2019. Т. 126. В. 3. С. 275. doi: 10.21883/OS.2019.03.47366.268-18; Bukvetskii B.V., Mirochnik A.G., Zhikhareva P.A. // Opt. Spectr. 2019. V. 126. N 3. P. 195-199. doi: 10.1134/S0030400X19030044

[16] Bruker, SMART and SAINT-Plus. Versions 5.0. Data Collection and Processing Software for the SMART System. Bruker AXS Inc. Madison, Wisconsin, USA. 1998.

[17] Sheldrick G.M. SHELXTL/PC. Versions 5.10. An Integrated System for Solving, Refining and Displaying Crystal Structures From Diffraction Data, Bruker AXS Inc., Madison, Wisconsin, USA. 1998.

\section{Конфликт интересов}

Авторы заявляют, что у них нет конфликта интересов. 